Research Article

\title{
pl6INK4/Ki-67 dual-staining expression as a prognostic indicator in laryngeal cancer
}

\begin{abstract}
Human Papilloma Virus (HPV) infection is thought to play a role in laryngeal carcinogenesis; particularly, p16 expression has been reported as a surrogate marker for high-risk HPV (HR-HPV) infection, associated with a subgroup of laryngeal cancers showing favourable prognosis. To evaluate HPV status, different detection molecular tests have been used and a large variation of HPV prevalence has been reported. The aim of the study was to detect HPV status by an immunohistochemical retrospective study conducted on seventy-three T1 N0 or carcinoma in situ (CIS) laryngeal biopsies, using a new established dual-staining test (p16/Ki-67), which might improve the specificity of the assay. Overall, 18 of 73 cases $(25 \%)$ were p16-positive: Verrucoid carcinomas showed p16 expression in almost all samples (87.5\%), whereas all G3 tumours were p16-negative. Patients with p16-negative tumours were more likely to relapse $(83 \%)$ than p16-positive cancers (17\%). Moreover, HPV genotyping detection was conducted on all 73 cases: $25 \%$ were classified as HPV+ and $75 \%$ were classified as HPV-; HPV+/p16+ cases were associated to HR-HPV whereas $\mathrm{HPV}+/ \mathrm{p} 16$ - samples (2 cases) were associated to LR-HPV. In consideration of these findings, investigation of p16 expression by the dual-staining test is sought as technically simple and potentially reliable assay for HR-HPV induced laryngeal cancers thus to be predictive of clinical outcome.
\end{abstract}

Keywords: p16; HPV; larynx, immunohisto chemistry, dual-staining
Volume I Issue 3 - 2014

\author{
Elisabetta Carico,' Marco Radici, ${ }^{2}$ Barbara \\ Bucci, ${ }^{3}$ Luca Firrisi, ${ }^{2}$ Alfredo Fabiano, ${ }^{4}$ \\ Gerardo Salerno,' Maria Rosaria Giovagnoli,' \\ Aldo Vecchione ${ }^{5}$ \\ 'Department of Clinical and Molecular Medicine, Sapienza \\ University, Italy \\ ${ }^{2}$ U.O.C. Otorhinolaryngology, S. Giovanni Calibita \\ Fatebenefratelli Hospital, Italy \\ ${ }^{3}$ Research Center San Pietro, Fatebenefratelli Hospital, Italy \\ ${ }^{4}$ S. Pathology, S. Giovanni Calibita Fatebenefratelli Hospital, Italy \\ ${ }^{5}$ Past Scientific Director G. Pascale National Institute for \\ Research and Treatment of Tumours, Italy
}

Correspondence: Elisabetta Carico, Department of Clinical and Molecular Medicine, Sapienza University, A.O. Sant'Andrea, Via di Grottarossa 1035, 00198 Rome, Italy, Tel 390633775345 , Fax390633775345, Email elisabetta.carico@uniromal.it

Received: October 09, 2014 | Published: October 30, 2014
Abbreviations: HPV, human papilloma virus; HR-HPV, high risk-HPV; LR-HPV, low risk HPV; CIS, carcinoma in situ; HNSCC, head and neck squamous cell carcinoma; PCR, polymerase chain reaction; DAB, 3,3' diaminobenzidine; ISH, in situ hybridization

\section{Introduction}

Among head and neck Squamous cell carcinomas (HNSCCs), the larynx is one of the sites most involved, being traditionally associated with tobacco and alcohol use, especially when in combination. ${ }^{1}$ As squamous carcinoma develops throughout a step progression in various stages of dysplasia, many molecular changes have been described. $^{2}$ The implication of Human Papilloma Virus (HPV) in HNSCC development, ${ }^{3,4}$ particularly, the infection with high-risk HPV types (HR-HPV) has also been etiologically linked with a subset of laryngeal carcinoma ${ }^{5}$ thus suggesting a different carcinogenetic pathway. Studies regarding HPV induced laryngeal cancers indicate that they are associated with improved outcomes, ${ }^{6}$ moreover they have more favorable prognosis when treated with surgery alone. At present, the causal role of HR-HPV in laryngeal carcinogenesis is controversial, mainly because no unanimously accepted detection method exists. Commonly used HPV detection methods for formalin fixed paraffin embedded tissue include: HPV consensus PCR method, type specific HPV detection by fluorescence in situ hybridization (FISH) and real-time PCR assays. Alternatively, the role of p16INK4a protein has been reported to identify HPV-related laryngeal cancers: ${ }^{8}$ hypermethylation and under-expression of p16 with loss of function is seen in most HNSCC; 9 however, loss of negative feedback on p16 expression mediated by $\mathrm{pRb}$ due to inactivation of $\mathrm{pRb}$ by HR-HPV E7 protein, leads to p16 over-expression in HR-HPV associated cancers. ${ }^{10}$ Different monoclonal antibody clones have been used to assess p16 expression, and different cut-off were employed to assess p16 positivity in HNSCC specimens. ${ }^{8,11-13}$ Recently, a novel biomarker concept, based on the combined detection of the p16 and Ki-67 biomarker protein expression has been designed: ${ }^{14}$ the simultaneous detection of p16, a cell-cycle regulatory protein, and the expression of a proliferation marker such as Ki-67 within the same epithelial cell may be interpreted as a surrogate marker of cell-cycle deregulation induced by HR-HPV infection. ${ }^{15}$

With the present study, using Immunohistochemistry assay in a series of 73 laryngeal biopsies with and without dysplasia, we aimed to identify the staining pattern of p16INK4a using a more objective immunohistochemical method for assessing HR-HPV. Such a biological marker for laryngeal carcinoma and its prognostic role were investigated whether they can be used as potential prognostic biomarkers in routine clinical practice.

\section{Materials and methods}

Seventy-three T1 N0 or carcinoma in situ (CIS) laryngeal biopsies were selected from the archives of the Fatebenefratelli Hospital from May 1992 through September 2004. All tissues were fixed in 10\% buffered formalin; hematoxylin and eosin-stained sections were examined at the light microscope level and graded for dysplasia according to the World Health Organization (WHO) classification ${ }^{16}$ as: G1 (33 cases), G2 (35 cases) and G3 (5 cases). Mean age of the patients at diagnosis was 62 years (range, 42 to 77 years); sex, smoking history, histology and tumor location are summarized in (Table 1). The patients had not received any preoperative therapy and surgery was the main mode of treatment given; clinical follow-up, ranging from 24 to 48 months, was conducted with a median followup of 36 months.

\section{pl6 assessment}

It was performed using a set of two different reagents $(\mathrm{mtm}$ Laboratories, Heidelberg): ${ }^{15}$ a primary monoclonal rabbit antibody clone 274-11AC3 directed against human $\mathrm{Ki}-67$ protein, and a 
primary monoclonal mouse antibody clone E6H4 directed to human p16INK4a protein. A two-step immunohistochemical assay was performed: after microwave antigen retrieval and endogenous peroxidases block, a cocktail of the primary antibodies was added for 30 minutes at R.T. Visualization reagents comprising a polymer reagent conjugated to horseradish peroxidases and goat anti-mouse antibody (15 minutes) and a polymer reagent conjugated to alkaline phosphatase and goat anti-rabbit antibody (15 minutes) were used. The samples were then developed with two different chromogenic reactions based on horseradish peroxidases-mediated conversion of a DAB chromogenic (10 minutes), and alkaline phosphatase-mediated conversion of Fast Red chromogenic (15 minutes) to visible reaction products at the respective antigen sites. A Squamous cell carcinoma of the cervix with known positive staining for p16INK4A was used as the positive control; an immunoglobulin class-matched no immune antibody was substituted for the primary antibody in the negative control. After counterstaining, a two-step mounting protocol was applied: in a first step an aqueous mounting medium, subsequently a permanent mounting medium were used. The results were evaluated by light microscopy. As the immunohistochemical procedure causes two different colored reaction products: a red staining of nuclei which indicates $\mathrm{Ki}-67$ expression and a brown precipitate at the p16INK4a antigen sites (cytoplasm and/or nuclei), the presence of $>10 \%$ of the epithelial cells with co localization of nuclear red staining and brown cytoplasmic staining was regarded as a positive test result. ${ }^{14} \mathrm{All}$ cases with dual-stain-positive cell (s) were reviewed by two independent pathologists to confirm the result.

\section{Detection of HPV DNA}

Five, $10-\mu \mathrm{m}$ sections of formalin-fixed, paraffin-embedded tissue from the same sixty-eight specimen blocks were transferred to eppendorf vials. The microtome blade was changed after each case. Human papillomavirus genotyping was performed by deparaffinization of the tissue sections and DNA extraction (Norgen's FFPE DNA Purification Kit), followed by HPV genotype detection using the INNO-LiPA HPV Genotyping Kit (Innogenetics). This assay permits specific detection of at least $25 \mathrm{HPV}$ genotypes (HPV types $6,11,16,18,31,33,35,39,40,42,43,44,45,51,52,53$, $.54,56,58,59,66,70,73$ and 74). After amplification of the HPV genome (L1 region), the PCR products were hybridized with specific oligonucleotide probes immobilized on a strip and detected using a chromogenic procedure following the manufacturer's protocol. DNA from the Caski cell line was used as a positive PCR control; PCR reagents lacking DNA served in each PCR amplification as a negative control.

\section{Statistical analysis}

A cross-tabulation analysis of p16INK4a immunohistochemical distribution with clinical and Histopathological findings was performed by the Chi-square test for trend or Fisher's exact test. The analysis of time to recurrence rates for p16INK4a positivity was calculated using the Kaplan-Meier method and compared using the log-rank test. Cox proportional hazards regression was performed to analyze the effect of all variables on recurrence times. A p value $<0.05$ was considered as statistically significant. Statistical analysis was performed using Graph Pad Prism 5.0 (GraphPad Software Inc., San Diego, CA, USA), SPSS V.18 (C Copyright IBM Corporation) and Sigma Plot V.1 1 (Copyright (C) 2008 Systat Software, Inc.).

\section{Results and discussion}

p16INK4a immunohistochemical distribution according to clinical and Histopathological findings is summarized in (Table 2). Overall, 18 of 73 cases $(25 \%)$ were positive for p16 expression, assessed by the dual-staining (p16/Ki-67) immunoreaction. Positive dual-staining immunoreactivity was found only in carcinomatous cells and was mainly observed in neoplastic nests (Figure 1).

Table I Clinical data

\begin{tabular}{llll}
\hline Patient/Tumour Data & Cases & $\%$ \\
\hline$N^{\circ}$ of patients & & 73 & \\
Mean age Range & & 62 & \\
Male & & $42-77$ & \\
Female & Never & 68 & 93.1 \\
Tobacco use & Former & 5 & 6.9 \\
& Current & 31 & 42.5 \\
Site & Supraglottic & 9 & 12.3 \\
& Glottic & 33 & 45.2 \\
Histology & CIS & 64 & 12.3 \\
& Squamous carcinoma & 3 & 87.7 \\
& Verrucoid carcinoma & 8 & 84.1 \\
Tumour differentiation & GI & 33 & 11.0 \\
& G2 & 35 & 45.2 \\
Follow-up & G3 & 5 & 68.0 \\
& NED & 67 & 92 \\
& Recurrences & 6 & 8 \\
\hline
\end{tabular}

CIS, carcinoma in situ; NED, no evident disease

Table 2 Correlation between pI6INK4 (dual-staining) immunohistochemical distribution and clinical data

\begin{tabular}{|c|c|c|c|c|}
\hline Factor & Cases & p I6/Ki-67 Positive Test & p I6/Ki-67 Negative Test & P value \\
\hline $\mathrm{N}^{\circ}$ of Patients & 73 & 18 (25\%) & $55(75 \%)$ & \\
\hline Tobacco Use & $\begin{array}{l}31 \text { never } \\
42 \text { current }+ \text { former }\end{array}$ & $\begin{array}{l}9(29 \%) \\
10(24 \%)\end{array}$ & $\begin{array}{l}22(71 \%) \\
32(66 \%)\end{array}$ & $0.78^{*}$ \\
\hline Site & $\begin{array}{l}64 \text { Glottic } \\
9 \text { Supraglottic }\end{array}$ & $\begin{array}{l}17(26 \%) \\
2(22 \%)\end{array}$ & $\begin{array}{l}47(73 \%) \\
7(78 \%)\end{array}$ & $1.00 *$ \\
\hline Histology & $\begin{array}{l}3 \text { CIS } \\
62 \text { Squamous carcinomas } \\
8 \text { Verrucoid carcinomas }\end{array}$ & $\begin{array}{l}0(0 \%) \\
12(19 \%) \\
7(87.5 \%)\end{array}$ & $\begin{array}{l}3(100 \%) \\
50(81 \%) \\
\mathrm{I}(12.5 \%)\end{array}$ & $<0.0001^{\wedge}$ \\
\hline Tumor Differentiation & $\begin{array}{l}33 \mathrm{GI} \\
35 \mathrm{G} 2 \\
5 \mathrm{G} 3\end{array}$ & $\begin{array}{l}12(36 \%) \\
7(20 \%) \\
0(0 \%)\end{array}$ & $\begin{array}{l}21(64 \%) \\
28(80 \%) \\
5(100 \%)\end{array}$ & $0.039^{\wedge}$ \\
\hline Recurrence & $\begin{array}{l}67 \mathrm{NO} \\
6 \mathrm{Yes}\end{array}$ & $\begin{array}{l}\text { I } 8(27 \%) \\
\text { I (17\%) }\end{array}$ & $\begin{array}{l}49(73 \%) \\
5(83 \%)\end{array}$ & $1.00 *$ \\
\hline
\end{tabular}

$\mathrm{CIS}$, carcinoma in situ

${ }^{\wedge}$ Fisher Test; $*$ Chi-square test for trend 
HPV genotyping detection was conducted on all 73 cases (Table 3): 18 samples (25\%) were classified as HPV+ and 55 samples (75\%) were classified as HPV-. Sixteen out of $18 \mathrm{HPV}+$ samples were associated to $\mathrm{p} 16$ expression and the genotyping assay demonstrated the presence of HR-HPV: 8 cases were typed as HPV-16 (50\%), 5 were typed as HPV-18 (31\%), 2 were typed as HPV $31(13 \%)$ and 1 was typed as HPV $52(6 \%)$. Fifty-three samples were HPV-/p16Two cases were HPV+/p16- and were associated to LR-HPV (HPV6). Two cases HPV-/p16+ were probably associated to a different HRHPV which was not present in the INNO LiPA assay.

Table 3 Correlation between HPV-DNA (INNO LiPA) and pI6INK4 (dualstaining) immunohistochemical status

\begin{tabular}{llll}
\hline HPV-DNA & pl6INK4 (dual-staining) Status & \\
\hline & Positive & Negative & Total \\
Positive & 16 & 2 & 18 \\
Negative & 2 & 53 & 55 \\
Total & 18 & 55 & 73 \\
\hline
\end{tabular}

Table 4 Multivariate Cox proportional hazards analysis for the time-torecurrence

\begin{tabular}{lll}
\hline Covariate & Beta & p-value \\
\hline Age & 0.745 & 0.464 \\
Sex & 0.371 & 0.274 \\
Tobacco Use & 0.59 & 0.672 \\
Grading & 0.382 & 0.439 \\
Histology & -0.348 & 0.774 \\
Site & -1.642 & 0.071 \\
pl6 & $\mathbf{I 7 . 0 4}$ & $\mathbf{0 . 0 4 2}$ \\
HPV-DNA & -0.126 & 0.452 \\
\hline
\end{tabular}

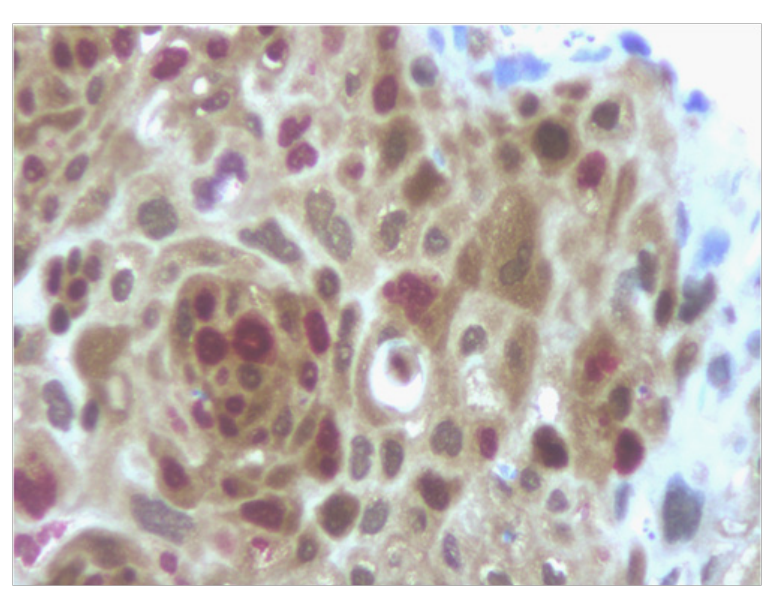

Figure I Detection of p 16 expression (brown cytoplasmic staining) and the additional staining for the $\mathrm{Ki}-67$ proliferation marker (nuclear red staining) within the same cell was regarded as a positive test result (immunoperoxidase, x400, original magnification).

P16 expression was therefore considered as an indicator of HRHPV presence and correlated to clinic pathological data. Regarding histological features, p16 was significant expressed in 36\% and $20 \%$ of G1 and G2 cases respectively, whereas all G3 tumors were p16negative $(\mathrm{p}=0.039)$; there was a significant higher $\mathrm{p} 16$ expression in Verrucoid carcinomas (87.5\%), whereas no CIS and $19 \%$ of Squamous carcinomas were $\mathrm{p} 16$-positive $(\mathrm{p}=<0.0001)$. The high $\mathrm{p} 16$ positivity regarding the Verrucoid histological variant allows stating the importance of this marker as a surrogate of HR-HPV infection, as the Verrucoid type is strictly associated to HPV infection. We also found a significant trend toward absent expression of p16 with increasing severity of grading as all G3 tumors were p16-negative; this might reflect the different biological behavior of HPV-related lesions being the decreased immunohistochemical expression of $\mathrm{p} 16$ predictive of poorly differentiated tumors.

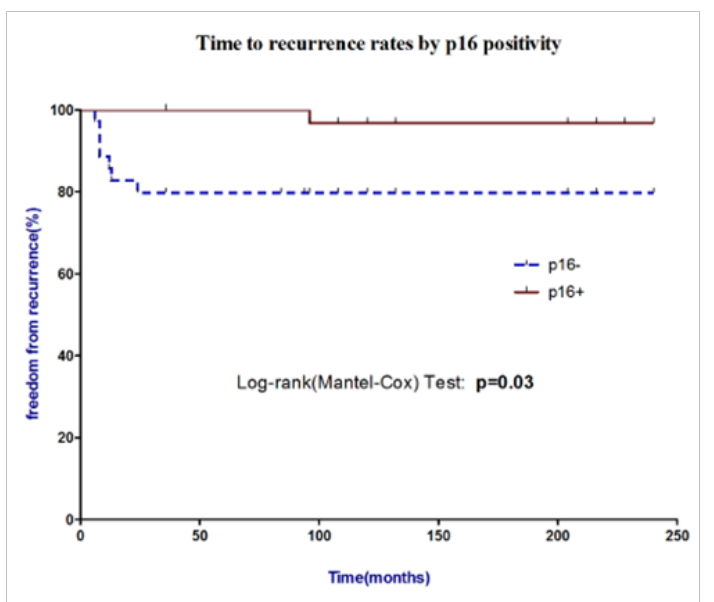

Figure 2 Time to recurrence rates by pl6 positivity. Log-rank (Mantel-Cox) curve showed that p 16 negative expression was found to have a significant effect on recurrence whereas a positive effect on outcome was evidenced in pl6 positive patients.

There was no significant correlation regarding tobacco consumption, as p16 expression was found in $29 \%$ and $24 \%$ samples from non smoking patients and former (defined as having quit smoking 5 years prior to diagnosis of laryngeal cancer) and current smokers, respectively. However, in this analysis the over-expression of p16 in $29 \%$ samples from non smokers, suggested the evidence of a causal role for HPV in carcinogenesis in patients without a history of tobacco use. No significant correlation was found between p16 expression and anatomical sites as p16 was expressed in $26 \%$ of Glottic carcinomas and $22 \%$ of Supraglottic cases, respectively.

During a median follow-up of 36 months, (range 24-48 months), the patients with p16-negative tumors were more likely to relapse (83\%) than patients with p16-positive cancers (17\%); log-rank (Mantel-Cox) curve showed that p16 negative expression was found to have a significant effect on recurrence whereas a positive effect on outcome was evidenced in $\mathrm{p} 16$ positive patients $(\mathrm{p}=0.03)$, (Figure 2 ). It has already been assessed that HPV-related HNCC lesions appear to be a distinct biological entity from those smoking and alcohol related as such lesions have different risk-factor profiles, respond far better to radiotherapy and have a more favorable prognosis; ${ }^{6}$ in this study the prognostic impact of the clinical, histological findings and p16 as a biological marker were evaluated by a multivariate Cox proportional hazards analysis for the time-to-recurrence and evidenced freedom from recurrence associated to p16 expression (0.042) as it might be considered as a surrogate marker of HR-HPV infection (Table 4).

\section{Conclusion}

The clinical significance of tumor HPV status in HNSCC is now well established; these tumors have been defined by HPV detection and belong to a subgroup which responds far better to radiotherapy than the smoking and alcohol related HNSCC. ${ }^{5}$ As reported by different Authors, ${ }^{17}$ the prevalence of HPV in laryngeal cancers varies significantly; methodological differences should be considered, as the assessment of HPV infection by in situ hybridization (ISH), polymerase chain reaction (PCR) by using the MY09/M11 amplification system or the GP5+/G6+ consensus primers, may lead to different results. 
Moreover, the presence of HPV does not necessarily imply its role in carcinogenesis, whereas the evidence of E6/E7 mRNA could better define the biological profile of the lesion. Recently, as a result from the expression of the HR-HPV related protein E7, p16INK4a positivity can be considered as a surrogate of HR-HPV infection; ${ }^{10}$ it has been currently established in HNSCC by using different monoclonal antibody clones, ${ }^{12,13,18,19}$ particularly, E6H4 monoclonal antibody has been employed and its reactivity has been assessed by different cut-off regarding the intensity of the immunoreaction, the staining pattern and the percentage of $\mathrm{p} 16$ positive atypical cells. ${ }^{5,8,19-23}$ As a consequence, the reported p16 positivity in HNSCC various significantly: Lassen et al. ${ }^{19}$ demonstrated p16 expression in $22 \%$ of HNSCC tumors whereas, according to a retrospective analysis performed by Ang et al..$^{22}$ p 16 expression was found in $96.1 \%$ of tumor samples. Regarding laryngeal cancers, an immunohistochemical study by Laco et al. ${ }^{24}$ demonstrated p16 over expression in $58 \%$ of neoplastic samples and Baumann et al. ${ }^{8}$ reported that all 5 cases that were HPV-positive by PCR, showed high levels of nuclear and cytoplasmic staining for $\mathrm{p} 16$, whereas 5 randomly selected HPV-negative cases did not.

With the present study we aimed to detect HPV status by an immunohistochemical study. This morphology independent biomarker approach, which recently has been assessed ${ }^{14,15}$ allowed us to found a $25 \%$ of 16 positivity in a retrospective study of T1 N0 or CIS laryngeal biopsies. Particularly, we analyzed p16 expression using dual-stain immunohistochemical localization for $\mathrm{p} 16 / \mathrm{Ki}$ 67 , which might enhance the specificity of the test as the scoring system is fundamentally different from the methods that have been previously used to score p14INK4. We utilized a highly validated assay that screens from a broad panel of HPV types (INNO-LiPA HPV Genotyping Kit) and found a correlation between HR-HPV and p16 expression status, thus we believe that p16 expression is a reliable biomarker of HPV infection in HNSCC.

The prognostic impact of p16 in laryngeal carcinoma was evaluated to consider whether such a biological marker can be used as a potential diagnostic tool. We postulate that p16 positivity might be regarded as a protective factor: log-rank (Mantel-Cox) curve showed that freedom from recurrence was worse among p16negative cases and in multivariate Cox proportional hazards analysis for the time-to-recurrence evidenced such an association. Previous studies demonstrated that over-expression of p16 assessed has been consistently related to HR-HPV infection it is easy to carry in paraffin embedded tissues and in fine-needle aspiration (FNA) of cytological specimens of cervical metastasis. Our findings confirm that p16 is of considerable diagnostic and prognostic value; moreover, the additional staining for Ki-67 might improve the reproducibility of the assay. Further evaluations of this biomarker could better identify the biological behavior and the clinical outcome of HR-HPV related HNSCC and could be taken into account for future therapeutic strategies.

\section{Acknowledgments}

The authors would like to thank Dr. Sandra Villani for technical assistance.

\section{Conflicts of interest}

Authors declare there are no conflicts of interest.

\section{References}

1. Le QT, Giaccia AJ. Therapeutic exploitation of the physiological and molecular genetic alterations in head and neck cancer. Clin Cancer Res. 2003;9(12):4287-4295.
2. Saunders JR. The genetic basis of head and neck carcinoma. Am J Surg. 1997;174(5):459-461.

3. Syrjanen S. Human papillomavirus (HPV) in head and neck cancer. $J$ Clin Virol. 2005;32(1):59-66.

4. Gillison ML, Lowy DR. A causal role for human papillomavirus in head and neck cancer. Lancet. 2014;363(9420):1488-1489.

5. Smeets SJ, Hesselink AT, Speel EJM, et al. A novel algorithm for reliable detection of human papillomavirus in paraffin embedded head and neck cancer specimen. Int J Cancer. 2007;121(11): 2465-2472.

6. Agudelo D, Quer $\mathrm{M}$, Leon $\mathrm{X}$, et al. Laryngeal carcinoma in patients without a history of tobacco and alcohol use. Head Neck. 1997;19(3):200-204.

7. Licitra L, Perrone F, Bossi P, et al. High-risk human papillomavirus affects prognosis in patients with surgically treated oropharyngeal Squamous cell carcinoma. J Clin Oncol. 2006;24(36):5630-5636.

8. Baumann JL, Cohen S, Evjen AN, et al. Human Papillomavirus in early laryngeal carcinoma. Laryngoscope. 2009;119(8):1531-1537.

9. Koscielny S, Dahse R, Ernst G, et al. The prognostic relevance of p16 Inactivation in head and neck cancer. ORL $J$ Otorhinolaryngol Relat Spec. 2007;69(1):30-36.

10. Vinokurova S, Wentzensen N, von Knebel Doeberitz M. Analysis of p16INK4a and integrated HPV genomes as progression markers. Methods Mol Med. 2005;119:73-83.

11. Yuen PW, Man M, Lam KY, et al. Clinicopathological significance of p16 gene expression in the surgical treatment of head and neck squamous cell carcinomas. J Clin Pathol. 2002;55(1):58-60.

12. Bradley KT, Budnick SD, Logani S. Immunohistochemical detection of p16INK4a in dysplastic lesions of the oral cavity. Mod Pathol. 2006;19(10):1310-1316

13. Gologan O, Barnes EL, Hunt JL. Potential diagnostic use of p16INK4A, a new marker that correlates with dysplasia in oral squamoproliferative lesions. Am J Surg Pathol. 2005;29(6):792-796.

14. Samarawardana P, Singh M, Shroyer KR. Dual stain immunohistochemical localization of p16INK4 and Ki-67: a synergistic approach to identify clinically significant cervical mucosal lesions. Appl Immunohistochem Mol Morphol. 2011;19(6):514-518.

15. Schmidt D, Bergeron C, Denton KJ, et al. p16/Ki-67 Dual-stain cytology in the triage of ASCUS and LSIL papanicolaou cytology: results from the European equivocal or mildly abnormal Papanicolaou cytology study. Cancer Cytopathol. 2011;119(3):158-166.

16. Shanmugaratnam K, Sobin LH. Histological typing of tumors of the upper respiratory tract and ear $2^{\text {nd }}$ ed. Springer-Verlag: Newyork, USA; 1991. $201 \mathrm{p}$

17. Torrente MC, Rodrigo JP, Haigentz M, et al. Human Papillomavirus infections in laryngeal cancer. Head Neck. 2011;33(4):581-586.

18. Cunningham LL, Pagano GM, Li M, et al. Overexpression of p16INK4 is a reliable marker of human papillomavirus-induced oral high-grade squamous dysplasia. Oral Surg Oral Med Oral Pathol Oral Radiol Endod. 2006;102(1):77-81.

19. Lassen P, Eriksen JG, Hamilton-Dutoit S, et al. Effect of HPV associated p16INK4a expression on response to radiotherapy and survival in squamous cell carcinoma of the head and neck. J Clin Oncol. 2009;27(12):1992-1998.

20. Kong CS, Narasimhan B, Cao H, et al. The relationship between human papillomavirus status and other molecular prognostic markers in head and neck squamous cell carcinomas. Int $J$ Radiat Oncol Biol Phys. 2009;74(2):553-561. 
21. Fischer CA, Zlobec I, Green E, et al. Is the improved prognosis of p16 positive oropharyngeal squamous cell carcinoma dependent of the treatment modality? Int J Cancer. 2010;126(5):1256-1262.

22. Ang KK, Harris J, Wheeler R, et al. Human papillomavirus and survival of patients with oropharyngeal cancer. NEngl J Med. 2010;363(1):24-35.
23. Lewis JS, Thorstad WL, Chernock RD, et al. p16 positive oropharyngeal squamous cell carcinoma: an entity with a favorable prognosis regardless of tumor HPV status. Am J Surg Pathol. 2010;34(8):1088-1096.

24. Laco J, Slaninka I, Jirasek M, et al. High-risk human papillomavirus infection and p16INK4a protein expression in laryngeal lesions. Pathol Res Pract. 2008;204(8):545-552. 